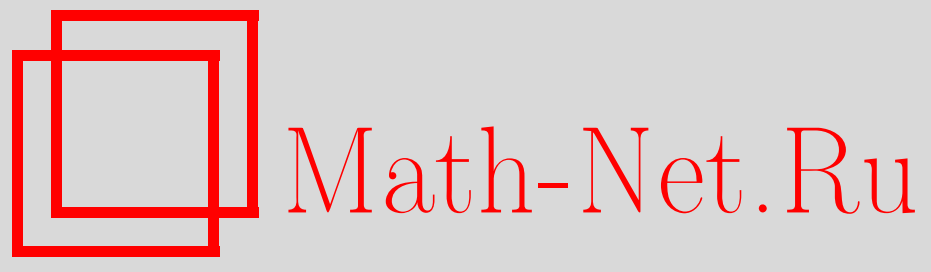

Б. С. Кашин, Об одном классе неравенств для ортонормированных систем, Матем. заметки, 2006, том 80, выпуск 2, 204-208

DOI: https://doi.org/10.4213/mzm2801

Использование Общероссийского математического портала Math-Net.Ru подразумевает, что вы прочитали и согласны с пользовательским соглашением http://www . mathnet.ru/rus/agreement

Параметры загрузки:

IP : 52.90 .164 .192

26 апреля 2023 г., 11:39:27

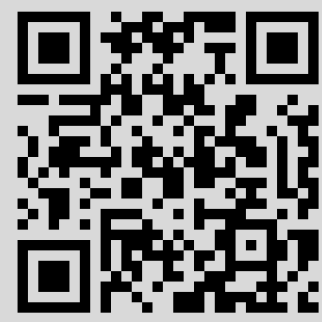




\section{ОБ ОДНОМ КЛАССЕ НЕРАВЕНСТВ ДЛЯ ОРТОНОРМИРОВАННЫХ СИСТЕМ}

\section{Б. С. Кашин}

В заметке предложен подход к доказательству неравенств типа Либа-Тирринга, использующий стандартный аппарат теории ортогональных рядов.

Библиография: 5 названий.

В 1976 г. в работе Либа и Тирринга [1] была установлена следующая

ТеоремА А. Для произвольной ортонормированной системъ $\Phi=\left\{\varphi_{j}\right\}_{j=1}^{N} \subset$ $L^{2}\left(\mathbb{R}^{d}\right)$ имеет место неравенство

$$
\left(\int_{\mathbb{R}^{d}} \rho_{\Phi}^{p /(p-1)} d x\right)^{2(p-1) / d} \leqslant C_{p, d} \sum_{j=1}^{N}\left\|\nabla \varphi_{j}\right\|_{2}^{2},
$$

если $\max (1, d / 2)<p \leqslant 1+d / 2$, әде

$$
\rho_{\Phi} \equiv \sum_{j=1}^{N} \varphi_{j}^{2}(x)
$$

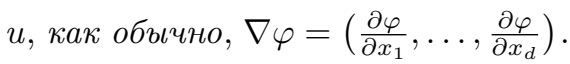

В дальнейшем серия неравенств типа (1) для конечных ортонормированных систем была установлена различными авторами. В частности, Ильиным [2] была доказана

Теорема В. Пусть $\left\{\varphi_{j}\right\}_{j=1}^{N} \subset L^{2}\left(S^{1}\right)$ - ортонормированная система функций, заданных на единичной окружности $S^{1}$, причем $\varphi_{j} \perp 1, j=1, \ldots, N$. Тогда имеет место неравенство

$$
\int_{S^{1}} \rho_{\Phi}^{2} d \mu \leqslant C \sum_{j=1}^{N}\left\|\varphi_{j}^{(1 / 2)}\right\|_{L^{2}\left(S^{1}\right)}^{2},
$$

где для $f(z)=\sum_{k \neq 0} \hat{f}(k) z^{k} \in L^{2}\left(S^{1}\right)$

$$
f^{(1 / 2)}=\sum_{k \neq 0}|k|^{1 / 2} \hat{f}(k) z^{k}
$$

- производная порядка $1 / 2$ от функции $f$, а $\mu$-нормированная мера Лебега на $S^{1}$.

Работа выполнена при финансовой поддержке Российского фонда фундаментальных исследований, грант № 05-01-00062.

(C) Б. С. КАшин, 2006 
Интерес к неравенствам типа Либа-Тирринга связан с их приложениями в теории дифференциальных уравнений с частными производными (см. подробнее [3], а также [2]). Известные методы доказательства этих неравенств основаны на нетривиальных результатах из спектральной теории, используют информацию о поведении отрицательных собственных значений операторов типа Шредингера (см., например, [2]). В этой заметке предлагается подход к неравенствам типа Либа-Тирринга, основанный на стандартном аппарате теории ортогональных рядов: неравенствах для случайных рядов и классической теореме Литлвуда-Пэли. Ниже мы продемонстрируем этот подход, установив некоторое обобщение теоремы В. При этом рассуждения, приведенные ниже, применимы, по всей видимости, и для доказательства других неравенств указанного типа. Кроме того, наш подход позволяет в определенной степени прояснить вопрос о том, для каких систем $\left\{\varphi_{j}\right\}$ неравенства типа Либа-Тирринга "точны по порядку".

Пусть

$$
\Phi=\left\{\varphi_{j}\right\}_{j=1}^{N} \subset L^{2}\left(S^{1}\right), \quad \text { причем } \quad \varphi_{j} \perp 1, \quad 1 \leqslant j \leqslant N .
$$

Определим оператор

$$
P_{\Phi}: l_{2}^{N} \rightarrow L^{2}\left(S^{1}\right)
$$

действующий по правилу:

$$
P_{\Phi}\left(\left\{c_{j}\right\}_{j=1}^{N}\right)=\sum_{j=1}^{N} c_{j} \varphi_{j}
$$

Пусть также при $\nu=0,1, \ldots$

$$
\pi_{\nu}: L^{2}\left(S^{1}\right) \rightarrow T_{2^{\nu}}
$$

- ортопроектор на пространство тригонометрических полиномов вида

$$
T_{2^{\nu}}=\left\{t(z): t=\sum_{2^{\nu} \leqslant|k|<2^{\nu+1}} a_{k} z^{k}\right\}
$$

и пусть

$$
\lambda_{\nu}(\Phi)=\left\|\pi_{\nu} \cdot P_{\Phi}: l_{2}^{N} \rightarrow T_{2^{\nu}}\right\|
$$

Очевидно, что $\lambda_{\nu} \leqslant 1, \nu=0,1, \ldots$, если $\Phi$ - ортонормированная (или субортонормированная) система. Поэтому следующее неравенство обобщает теорему В.

Теорема 1. Для любой системы действительных функций $\Phi$ вида (3)

$$
\int_{S^{1}} \rho_{\Phi}^{2} d \mu \leqslant C \sum_{\nu=0}^{\infty} \gamma_{\nu}(\Phi) \cdot \sum_{j=1}^{N} \sum_{2^{\nu} \leqslant|k|<2^{\nu+1}}\left|\widehat{\varphi}_{j}(k)\right|^{2},
$$

где при $\nu=0,1,2, \ldots$

$$
\gamma_{\nu}(\Phi)=\sum_{\beta=0}^{\nu} 2^{\beta} \cdot \lambda_{\beta}^{2}(\Phi)
$$

функиия $\rho_{\Phi}$ определена в (2), числа $\lambda_{\beta}(\Phi)$ в (4), а C - абсолютная постоянная. 
Прежде чем перейти к доказательству теоремы 1, напомним некоторые нужные нам факты.

Пусть $1 \leqslant p<\infty$ и $\varphi_{j} \in L^{p}\left(S^{1}\right), 1 \leqslant j \leqslant N$. Тогда, положив $\mathscr{F}=\left(\sum \varphi_{j}^{2}\right)^{1 / 2}$, имеем (см. [4; с. 52]):

$$
C_{1}(p)\|\mathscr{F}\|_{L^{p}\left(S^{1}\right)}^{p} \leqslant \int_{S^{1}}\left\|\sum_{j=1}^{N} r_{j}(t) \varphi_{j}\right\|_{L^{p}\left(S^{1}\right)}^{p} d t \leqslant C_{2}(p)\|\mathscr{F}\|_{L^{p}\left(S^{1}\right)}^{p},
$$

где $\left\{r_{j}(t)\right\}$ - функции Радемахера, $C_{1}(p)>0$.

Далее нам потребуется известное следствие теоремы Литлвуда-Пэли (см., например, [5; с. 56]): если, принимающая действительные значения, функция $f \in L^{p}\left(S^{1}\right)$, $1<p<\infty, f \perp 1$ и

$$
f=\sum_{\nu=0}^{\infty} \delta_{\nu}(f), \quad \delta_{\nu}(f)=\sum_{2^{\nu} \leqslant|k|<2^{\nu+1}} \hat{f}(k) z^{k}
$$

то

$$
C_{3}(p)\|f\|_{L^{p}\left(S^{1}\right)} \leqslant\left\|\left(\sum \delta_{\nu}^{2}\right)^{1 / 2}\right\|_{L^{p}\left(S^{1}\right)} \leqslant C_{4}(p)\|f\|_{L^{p}\left(S^{1}\right)}, \quad C_{3}(p)>0 .
$$

Нам потребуется также следующая простая

Лемма. Для любой системы функиий $\Phi$ вида (3) при $\beta=0,1, \ldots$ справедливо неравенство

$$
A=\left\|\sum_{j=1}^{N} \delta_{\beta}^{2}\left(\varphi_{j}\right)\right\|_{L^{\infty}\left(S^{1}\right)} \leqslant 2^{\beta+1} \cdot \lambda_{\beta}^{2}
$$

Действительно, пусть $z_{0} \in S^{1}-$ такая точка, что

$$
\left(\sum_{j=1}^{N} \delta_{\beta}^{2}\left(\varphi_{j}\right)\left(z_{0}\right)\right)^{1 / 2}=A^{1 / 2}
$$

Найдется набор чисел $\left\{c_{j}\right\}_{j=1}^{N}$ с $\sum_{j=1}^{N} c_{j}^{2}=1$ такой, что для тригонометрического многочлена

$$
\sum_{j=1}^{N} c_{j} \delta_{\beta}\left(\varphi_{j}\right)=P(z)=\sum_{2^{\beta} \leqslant|k|<2^{\beta+1}} a_{k} z^{k}
$$

имеют место неравенства

$$
\|P\|_{L^{\infty}\left(S^{1}\right)} \geqslant\left|P\left(z_{0}\right)\right|=A^{1 / 2}
$$

а значит (см. (4)),

$$
\begin{aligned}
A^{1 / 2} & \leqslant\|P\|_{L^{\infty}\left(S^{1}\right)} \leqslant \sum_{2^{\beta} \leqslant|k|<2^{\beta+1}}\left|a_{k}\right| \leqslant 2^{(\beta+1) / 2}\left(\sum_{2^{\beta} \leqslant|k|<2^{\beta+1}}\left|a_{k}\right|^{2}\right)^{1 / 2} \\
& =2^{(\beta+1) / 2}\|P\|_{L^{2}\left(S^{1}\right)} \leqslant 2^{(\beta+1) / 2} \lambda_{\beta},
\end{aligned}
$$

что и требовалось (фактически мы использовали неравенство Никольского разных метрик, сводящееся в данном случае к неравенству Коши). 
ДоКАЗАТЕЛЬСТво тЕОРЕмЫ 1. Используя (6), а затем (7) и вводя обозначение $\delta_{\nu}(t, z)=\delta_{\nu}\left(\sum_{j=1}^{N} r_{j}(t) \varphi_{j}(z)\right)$, имеем

$$
\begin{aligned}
Q \equiv \int_{S^{1}} \rho_{\Phi}^{2} d \mu & \leqslant C_{5} \int_{0}^{1}\left\|\sum r_{j}(t) \varphi_{j}\right\|_{L^{4}}^{4} d t \\
& \leqslant C_{6} \int_{0}^{1} \int_{S^{1}}\left[\sum_{\nu=0}^{\infty} \delta_{\nu}^{2}(t, z)\right]^{2} d \mu d t .
\end{aligned}
$$

Следовательно

$$
\frac{Q}{C_{6}} \leqslant \sum_{\nu, \beta=0}^{\infty} \int_{0}^{1} \int_{S^{1}} \delta_{\nu}^{2}(t, z) \delta_{\beta}^{2}(t, z) d \mu d t
$$

При этом

$$
\delta_{\nu}^{2}(t, z)=\left(\sum_{j=1}^{N} r_{j}(t) \delta_{\nu}\left(\varphi_{j}\right)(z)\right)^{2}=\sum_{j, q=1}^{N} r_{j}(t) r_{q}(t) \delta_{\nu}\left(\varphi_{j}\right) \delta_{\nu}\left(\varphi_{q}\right)
$$

а потому для каждого $t$

$$
\begin{aligned}
\int_{S^{1}} \delta_{\nu}^{2}(t, z) \delta_{\beta}^{2}(t, z) d \mu= & \sum_{j, q, h, s=1}^{N} r_{j}(t) r_{q}(t) r_{h}(t) r_{s}(t) \\
& \times \int_{S^{1}} \delta_{\nu}\left(\varphi_{j}\right) \delta_{\nu}\left(\varphi_{q}\right) \delta_{\beta}\left(\varphi_{h}\right) \delta_{\beta}\left(\varphi_{s}\right) d \mu .
\end{aligned}
$$

Учитывая, что интеграл $\int r_{j} r_{q} r_{h} r_{s} d t \neq 0$, только если в наборе $(j, q, h, s)$ каждое число встречается четное число раз (и тогда этот интеграл равен единице), мы находим

$$
\begin{aligned}
\mathrm{I}_{\nu, \beta} \equiv & \int_{0}^{1} \int_{S^{1}} \delta_{\nu}^{2}(t, z) \delta_{\beta}^{2}(t, z) d \mu d t=\sum_{j=1}^{N} \sum_{h=1}^{N} \int_{S^{1}} \delta_{\nu}^{2}\left(\varphi_{j}\right) \delta_{\beta}^{2}\left(\varphi_{h}\right) d \mu \\
& +2 \sum_{j=1}^{N} \sum_{q=1}^{N} \int_{S^{1}} \delta_{\nu}\left(\varphi_{j}\right) \delta_{\beta}\left(\varphi_{j}\right) \delta_{\nu}\left(\varphi_{q}\right) \delta_{\beta}\left(\varphi_{q}\right) d \mu-2 \sum_{j=1}^{N} \int_{S^{1}} \delta_{\nu}^{2}\left(\varphi_{j}\right) \delta_{\beta}^{2}\left(\varphi_{j}\right) d \mu \\
\leqslant & \int_{S^{1}} \sum_{j=1}^{N} \delta_{\nu}^{2}\left(\varphi_{j}\right) \cdot \sum_{j=1}^{N} \delta_{\beta}^{2}\left(\varphi_{j}\right) d \mu+2 \int_{S^{1}}\left[\sum_{j=1}^{N} \delta_{\nu}\left(\varphi_{j}\right) \delta_{\beta}\left(\varphi_{j}\right)\right]^{2} d \mu .
\end{aligned}
$$

Применяя неравенство Коши к сумме в последнем интеграле, находим

$$
\mathrm{I}_{\nu, \beta} \leqslant 3 J_{\nu, \beta}, \quad \text { где } \quad J_{\nu, \beta} \equiv \int_{S^{1}} \sum_{j=1}^{N} \delta_{\nu}^{2}\left(\varphi_{j}\right) \cdot \sum_{j=1}^{N} \delta_{\beta}^{2}\left(\varphi_{j}\right) d \mu .
$$

Поэтому (см. (8))

$$
\frac{Q}{C_{6}} \leqslant 3 \sum_{\nu, \beta=0}^{\infty} J_{\nu, \beta} \leqslant 6 \sum_{\nu, \beta: \beta \leqslant \nu} J_{\nu, \beta}
$$


Используя для оценки слагаемых в сумме (9) лемму, имеем (см. также (5))

$$
\frac{Q}{C_{6}} \leqslant 6 \sum_{\nu=0}^{\infty}\left[\sum_{\beta=0}^{\nu} 2^{\beta+1} \lambda_{\beta}^{2}\right] \cdot \int_{S^{1}} \sum_{j=1}^{N} \delta_{\nu}^{2}\left(\varphi_{j}\right) d \mu=12 \sum_{\nu=0}^{\infty} \gamma_{\nu}(\Phi)\left[\sum_{j=1}^{N} \sum_{2^{k} \leqslant|k|<2^{k+1}}\left|\widehat{\varphi}_{j}(k)\right|^{2}\right] .
$$

Теорема 1 доказана.

\section{СПИСОК ЦИТИРОВАННОЙ ЛИТЕРАТУРЫ}

[1] E. Lieb, W. Thirring, "Inequalities for the moments of the eigenvalues of the Schrödinger hamiltonian and their relation to Sobolev inequalities", Studies in Mathematical Physics, Essays in honor of Valentine Bargmann, Princeton Univ. Press, Princeton, 1976, 269-303.

[2] А. А. Ильин, "Интегральные неравенства Либа-Тирринга и их приложения к аттракторам уравнений Навье-Стокса", Матем. сб., 196:1 (2005), 33-66.

[3] R. Temam, Infinite-Dimensional Dynamical Systems in Mechanics and Physics, SpringerVerlag, New York, 1997.

[4] Б. С. Кашин, А. А. Саакян, Ортогоналъные ряды, АФЦ, М., 1999.

[5] С. М. Никольский, Приближение функиий многих переменных и теоремы вложения, Физматлит, М., 1977.

\section{Б. С. Кашин}

Поступило

Математический институт им. В. А. Стеклова РАН 